\title{
Pseudo-Bayesian and Bayesian Approach to Auditing
}

\author{
R. W. ANDREWS $†$ and T. M. F. SMITH $\ddagger$ \\ †University of Michigan; $\ddagger$ University of Southampton
}

\section{Introduction}

Previous papers in this conference have examined problems in medicine, law, insurance and education. In this paper we examine auditing, an important part of the work of accountants. It is no coincidence that these applications are to "the professions". Professional people are paid to make judgements and these judgements are usually based on a variety of forms of evidence few of which are quantifiable within a frequentist approach to statistics. For the Bayesian, however, qualitative evidence can be incorporated, albeit with difficulty, into a subjective distribution and hence can add a scientific dimension to some of the mysteries of professional judgement.

The problem in auditing is to combine qualitative evidence based on observation of the accounting system and enquiries as to its working with quantitative evidence based on the results of checking specific items for compliance with the system and for numerical accuracy. The overall objective is for the auditor to attest to the accuracy of the numbers presented in the final accounts such as those in the year end balance sheet.

\section{Sources of evidence}

It is possible to identify four distinct sources of evidence upon which the auditor bases his opinion.

(i) System evaluation ( $S E)$

This is qualitative evidence about the effectiveness of the accounting system. Particular attention is paid to the internal control system which is set up to prevent errors from being included in the accounts and to detect and correct errors that are included.

\section{(ii) Compliance testing (CT)}

This gives quantitative evidence about how effectively the system works. Sometimes individual items are followed through the system to see if all the appropriate checks are being carried out. However, in most cases compliance testing takes the form of sampling a key control to see if it is being properly applied. Errors may be of many types ranging from the failure to implement one or more controls to an actual recording error in a money value. If controls are not being applied then the system is not being complied with and hence is more likely to produce monetary errors.

(iii) Substantive tests of details $(T D)$

These are direct observations on the accuracy of the recorded values of a sample of indi- 
vidual items drawn from the accounts. These tests provide direct evidence about the accuracy of the totals. The statistical problem is that usually there are few errors in recorded money values and so inference about the total value of error is based on very little error data.

\section{(iv) Analytical review $(A R)$}

This is qualitative evidence about the totals or balances. Balances for this year may be compared with those for previous years and with those in similar firms or industries. Various financial ratios are calculated and sometimes regression analysis is employed.

The decisions facing the auditor relate to the nature, timing and extent of the quantitative tests, CT and TD. A major problem is how to incorporate the valuable information collected in SE and AR into these decisions. Classical frequentist inferences are often used but then $\mathrm{SE}$ and $\mathrm{AR}$ have to be incorporated in an ad hoc manner.

\section{A pseudo-Bayes approach}

Auditors are aware of the need to combine qualitative and quantitative evidence in forming their opinions. In the USA this has been recognized formally in Statement on Auditing Standards No. 39 (1981), which introduces the idea of ultimate risk. Ultimate risk is "a combination of the risk that material errors will occur in the accounting process used to develop the financial statements and the risk that any material errors that do occur will not be detected by the auditor. The risk of these adverse events occurring jointly can be viewed as the product of the respective individual risks.... Ultimate risk includes both uncertainties due to sampling and uncertainties due to factors other than sampling."

If we let $U$ be the ultimate risk, $S$ be the system risk and $T$ be the testing (sampling) risk, then the above rule gives

$$
U=S \times T \text {. }
$$

The auditor uses professional judgement to set $U$ at say 0.01 . If $S$ is assessed at $0 \cdot 20$ then this makes $T=0 \cdot 05$. This sets the level for a significance test or confidence interval. If the system is very good and $S=0.01$ then this makes $T=1.00$, which means, based on this model, that no testing is required. On the other hand if the system is very bad and $S \simeq 1.00$ then $T=0.01$ and $U$ is the same as the testing risk. Although the events to which these risks apply are not clearly defined the ensuing rules are sensible and lead to the decision that small samples will suffice when the system is good but large samples are needed when it is bad. The frequentist position finds it hard to accommodate such arguments.

In practice auditors often use the above procedure to determine the level of the Type II error in a significance test and since significance tests are not coherent (see e.g. Lindley, 1972) the above approach is pseudo-Bayesian rather than Bayesian.

\section{A Bayesian approach}

In a Bayesian approach it is necessary to consider the order in which evidence is acquired and the mechanisms that can generate errors. We assume that $S E$ is evaluated first followed by the test data CT and TD and finally AR. Letting $E$ denote the unknown total money value of the error in the balance being examined the object is to find the posterior probability of $E$, which is given by

$$
\begin{aligned}
& p(E \mid \mathrm{SE}, \mathrm{CT}, \mathrm{TD}, \mathrm{AR}) \\
& \propto p(E \mid \mathrm{SE}) p(\mathrm{CT} \mid E, \mathrm{SE}) p(\mathrm{TD} \mid E, \mathrm{SE}, \mathrm{CT}) p(\mathrm{AR} \mid E, \mathrm{SE}, \mathrm{CT}, \mathrm{TD})
\end{aligned}
$$


The next problem is to model the various components of this posterior distribution.

The distribution $p(E \mid \mathrm{SE})$ has to be evaluated subjectively. A common procedure is to produce a final score based on scores for various components of the system. This score must be translated into a distribution that captures the auditors uncertainty.

For CT we adopt an approach due to the accounting firm Thompson-McLintock and break down the total compliance error into component parts. This reliability theory approach leads to $p(C T \mid E, S E)$. For the term TD we adopt the Bayesian approach of Cox and Snell (1979) (see also Smith, 1979; Moors, 1982), in which the probability that an item has an error of a given amount is viewed as the probability of an error multiplied by the conditional probability of the size of the error given that an error has occurred. This model is identical in structure to that of Phillips and Wisniewski (1983). It should be noted that to accountants D. R. Cox is one of the leading Bayesians! A similar approach has been adopted by Kaplan (1973) and Gartska (1977). The results above can be combined to give $p(E \mid \mathrm{SE}, \mathrm{CT}, \mathrm{TD})$. The evidence from AR is then assessed and combined with $p(E \mid \mathrm{SE}, \mathrm{CT}, \mathrm{TD})$ to give the final opinion about the financial statement under examination. The full details of the analysis appear in Andrews and Smith (1982).

\section{References}

Andrews, R. W. and Smith, T. M. F. (1982). A Bayesian approach to the audit process. Typescript submitted for publication, July 1982.

Cox, D. R. and Snell, E. J. (1979). On sampling and the estimation of rare errors. Biometrika, 66 (1), 125-33.

Gartska, S. J. (1977). Models fer computing upper error limits in dollar unit sampling. Accounting Review, 179-92.

Kaplan, R. S. (1973). A stochastic model for auditing. Journal of Accounting Research (Spring 1973a), 38-46. Lindley, D. V. (1972). Bayesian Statistics, a review. S.I.A.M. monograph, Philadelphia.

Moors, J. J. A. (1982). Bayes estimation in sampling for auditing. Statistician.

Phillips, L. D. and Wisniewski, T. K. (1983). Bayesian models for computer-aided underwriting. Statistician, 32 (1), 252-263.

Smith, T. M. F. (1979). Statistical sampling in auditing: a statistician's viewpoint. Statistician, 28 (4), 267-80.

Statement of auditing standards, No. 39 (1981). American Institute of Certified Public Accountants, Inc., New York. 